\title{
Nuclear Photoproduction of Pseudoscalar Mesons at Forward Angles up to 6.0 GeV
}

\author{
T.E. Rodrigues ${ }^{1}$, J.D.T. Arruda-Neto ${ }^{1}$, J. Mesa ${ }^{2}$, C. Garcia ${ }^{3}$, K. Shtejer ${ }^{1,3}$, \\ D. Dale ${ }^{4}$ I. Nakagawa ${ }^{5}$ and P.L. Cole ${ }^{4}$ \\ ${ }^{1}$ Physics Institute, University of São Paulo, São Paulo,Brazil (tulio@if.usp.br) \\ ${ }^{2}$ Department of Physics and Biophysics - UNESP, Botucatu, Brazil \\ ${ }^{3}$ Center of Applied Studies for Nuclear Developments, Havana, Cuba \\ ${ }^{4}$ Department of Physics, Idaho State University, Pocatello ID, 83209, USA \\ ${ }^{5}$ RIKEN 2-1 Hirosawa, Wako, Saitama 351-0198, Japan
}

\begin{abstract}
The nuclear incoherent $\pi^{0}$ photoproduction cross section from ${ }^{12} \mathrm{C}$ is evaluated at forward angles in the 4.0 to $6.0 \mathrm{GeV}$ energy range using the multicollisional intranuclear cascade model MCMC. The model incorporates some improvements in comparison with previous versions associated with the momentum distribution (MD) for light nuclei - extracted from the available (e,e'p) data - as well as the evaluation of the shadowing effects during the photonucleus interaction. The final results of the single and double differential cross sections at forward angles are very sensitive to the MD parameterizations due to the Pauli principle, which largely suppresses the cross sections for low momentum transfer. The attenuation of the nuclear cross section due to pion - nucleus final state interactions is approximately $40 \%$ (without nuclear shadowing), which is in nice agreement with the predictions from the Glauber model. The single and double $\pi^{0}$ differential cross sections are presented for possible applications for the interpretation of the inelastic background in the PrimEx experiment at the Jefferson Laboratory.
\end{abstract}

Keywords: Meson photoproduction, intranuclear cascade model, nuclear matter, meson-nucleus final state interactions, nuclear shadowing

PACS: 24.10.Lx, 25.20.Lj, 13.40.Hq, 12.40.Vv

\section{INTRODUCTION}

The advent of the PrimEx Collaboration at the Jefferson Laboratory ${ }^{1}-$ which is currently proposing a precision measurement of the $\pi^{0}-\rightarrow \gamma \gamma$ decay width by fitting the $\pi^{0}$ differential cross section at forward angles - has brought a renewed interest for calculations of the nuclear incoherent cross section for $\pi^{0}$ photoproduction from complex nuclei. This incoherent mechanism is expected to contribute significantly at forward angles also in the quasi-elastic domain, where the energy carried out by the pion is very close to the photon energy. Such a result requires a complete understanding of both the angular distributions of the produced pions and the double differential cross sections in order to disentangle the relative contributions of the competing mechanisms using information about the pion spectra.

In a previous work ${ }^{2}$ we have developed an extended version of the Multicollisional Monte Carlo intranuclear cascade model $(\mathrm{MCMC})^{3,4}$ for the interpretation of the recent data of incoherent $\pi^{0}$ photoproduction near the Delta region for ${ }^{12} \mathrm{C}$ and ${ }^{208} \mathrm{~Pb}$

CP947, VII Latin American Symposium on Nuclear Physics and Applications

edited by R. Alarcon, P. L. Cole, C. Djalali, and F. Umeres

(C) 2007 American Institute of Physics 978-0-7354-0461-8/07/\$23.00 
obtained at the Mainz Microton Collaboration (MAMI) ${ }^{5}$. The cross sections were satisfactorily reproduced assuming that the $\gamma N$ coupling in the $s$-channel is largely dominated by a Delta isobar (1232) excitation, with subsequent decay into the $\pi^{0} \mathrm{~N}$ channel. In this work, we have also presented some predictions for the $\pi^{0}$ incoherent photoproduction cross sections for ${ }^{12} \mathrm{C}$ and ${ }^{208} \mathrm{~Pb}$ within the range from 4.0 to $6.0 \mathrm{GeV}$, neglecting shadowing effects of the incoming photon. Coherent production (electromagnetic/nuclear) of neutral pions was also investigated in a recent paper taking into account the relativistic recoil of the nucleus. The calculations of the pion spectra due to coherent production showed that those mechanisms are largely concentrated within the quasi-elastic peak, with the total energy of the mesons being typically within the photon energy $k$ and $k-10 \mathrm{MeV}$.

The MCMC model was also recently applied for the calculation of the $\eta$ photoproduction cross sections in $\mathrm{Be}$ and $\mathrm{Cu}$ around $9.0 \mathrm{GeV}^{7}$, reproducing quite successfully the inelastic background in the photoproduction yields obtained at Cornell ${ }^{8}$.

In this work, we report improved calculations for the incoherent $\pi^{0}$ photoproduction cross sections on ${ }^{12} \mathrm{C}$ taking into account the shadowing effects of the incoming photon, as well as accurate momentum distributions of the bound nucleons obtained via the analysis of the $1-s$ and $1-p$ proton knock-out data from ${ }^{12} \mathrm{C}(e, e, p){ }^{11} \mathrm{~B}$ experiments.

\section{INCOHERENT PHOTOPRODUCTION OF NEUTRAL PIONS}

\section{Momentum Distribution of the Bound Nucleons of ${ }^{12} \mathrm{C}$}

The momentum distribution (MD) of the bound nucleons in light nuclei consists of an important ingredient for calculations in a low momentum transfer regime, since the Pauli-blocking mechanism is expected to play a major role. Small differences in the MD change considerably the kinematics of the photon-nucleon interaction and these changes reflect into the angular distributions of the produced mesons. For this purpose, we have investigated several parameterizations of the MD based on the current $1-s$ and 1-p proton knock-out data from the reaction ${ }^{12} \mathrm{C}\left(e, e^{\prime} p\right)^{11} \mathrm{~B}$. Table 1 summarizes the world data used in the calculations.

The reduced cross sections obtained in the experiments sketched in Table 1 are the spectral functions of ${ }^{12} \mathrm{C}$ integrated in different ranges of missing energies and represent the momentum distribution of the bound nucleons taking into account the Final State Interactions (FSI) of the emitted proton and electron on their way out of the nucleus. For this reason, these distributions are interpreted as the distorted momentum distributions, where the distortion effects depend very critically upon the reaction mechanism. However, for the evaluation of the ${ }^{12} \mathrm{C}$ structure - which is the crucial step in the present analysis - one has to consider the "true" MD, instead of the distorted. The undistorted MD for the $s$ and $p$-shells were calculated in the Plane Wave Impulse Approximation (PWIA) in ref. 9 (Figure 1) and will be used as a reference 
value for this analysis. A complete review of the MD and the nuclear transparency of

${ }^{12} \mathrm{C}$ for proton knock-out reactions can be found elsewhere ${ }^{9}$.

\begin{tabular}{|c|c|c|c|c|c|c|}
\hline \multirow{6}{*}{ 1-s knock-out } & Dataset & $\Delta E_{m}(\mathrm{MeV})$ & $T_{p}(\mathrm{MeV})$ & $S_{l p} / S_{1 s}$ & Kinematics & $Q^{2}(\mathrm{GeV} / c)^{2}$ \\
\hline & Saclay $76^{(10)}$ & $15-22$ & 87 & $2.19(13)$ & perpendicular & 0.16 \\
\hline & Saclay $81^{(11)}$ & $15-22$ & 99 & $2.28(7)$ & parallel & $0.09-0.32$ \\
\hline & Saclay $81^{(11)}$ & $15-22$ & 99 & $2.31(6)$ & perpendicular & $0.09-0.32$ \\
\hline & Tokyo $76^{(12)}$ & $6-30$ & 159 & $2.16(10)$ & perpendicular & 0.29 \\
\hline & NIKHEF $88^{(13)}$ & G.S. & 70 & $2.20(4)$ & parallel & $0.02-0.26$ \\
\hline \multirow{3}{*}{ 1-p knock-out } & Saclay $76^{(10)}$ & $30-50$ & 87 & $0.84(2)$ & perpendicular & 0.16 \\
\hline & Tokyo $76^{(12)}$ & $21-66$ & 136 & $1.19(5)$ & perpendicular & 0.29 \\
\hline & NIKHEF $88^{(13)}$ & $30-39$ & 70 & $0.047(2)$ & parallel & $0.02-0.26$ \\
\hline
\end{tabular}

The momentum distributions associated with the data from Table 1 were fitted separately in order to provide empirical information about the sensitivity of the cascade model with small changes in these observables. A typical fitting of a specific dataset from the NIKHEF experiment ${ }^{13}$ is presented in Figure 2.

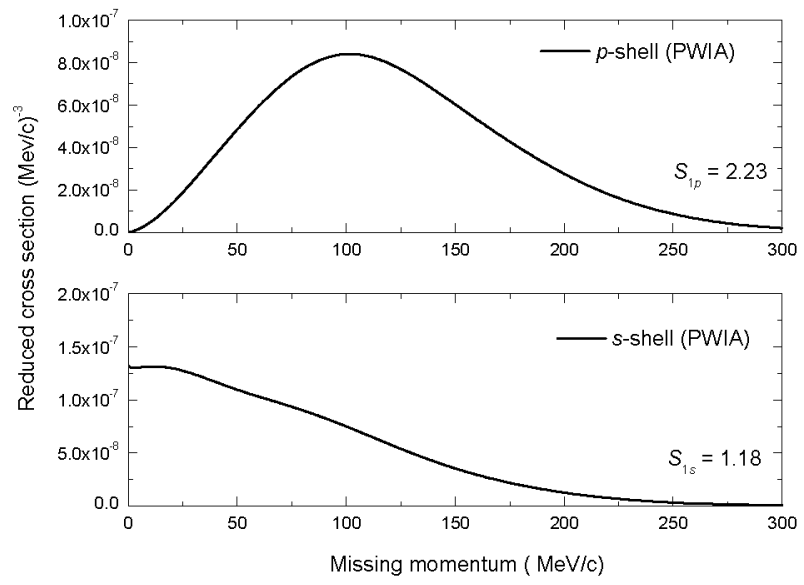

FIGURE 1. Momentum distributions (reduced cross sections) in the PWIA for 1 proton knock-out from the $s$ and $p$-shells of ${ }^{12} \mathrm{C}$ (Ref. 9).

So, the calculations outlined in this work were carried out using the MD in the PWIA (Figure 1), as well as the combinations of empirical distributions extracted from the data of Table 1 . The relative variation of the cross sections obtained in different runs with different $\mathrm{MD}$ parameterizations provided the estimated systematic uncertainty of the MCMC model with this input. It is worth mentioning, however, that the label PWIA appears in two different cases in the paper, namely: the $\mathrm{MD}$ parameterization and the total $\pi^{0}$ production cross section (see next section). The later is simply the nucleus mass times the elementary cross section from a single nucleon but is also calculated using the PWIA for the MD. 


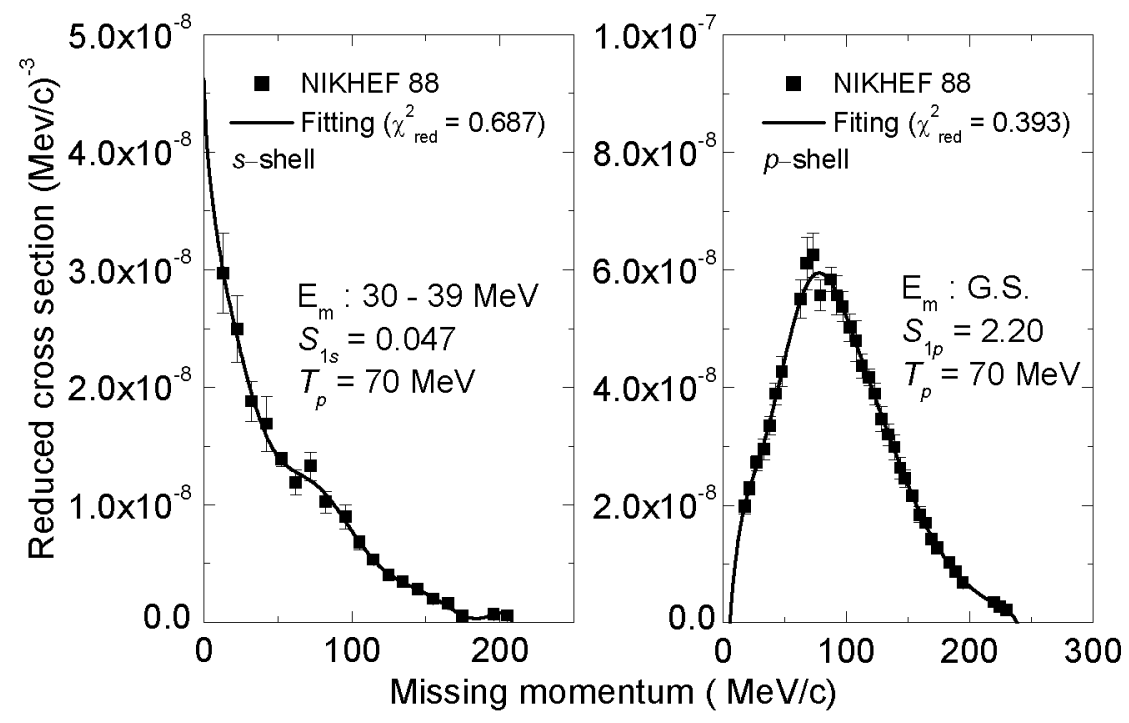

FIGURE 2. Fitted momentum distributions for the $s$ and $p$-shells of ${ }^{12} \mathrm{C}$ (solid lines). The data points were taken from ref. 13.

\section{Shadowing Effects in High Energy Photo-Nucleus Interactions}

The VMD model predicts a shadowing effect on the nuclear photoabsorption cross section due to the direct coupling of photons (real or virtual) with vector mesons, which are strongly absorbed deep in the nuclei ${ }^{14}$. Such attenuation of the vector meson can be obtained experimentally by measuring the ratio between the total hadronic cross section for complex nuclei $\sigma_{\gamma 4}$ and from a single nucleon $\sigma_{\not N}$ (averaged over proton and neutron amplitudes). In other words, one can verify experimentally that $\sigma_{\gamma A}$ $<A \sigma_{\not N}$, which is a clear evidence that the physical photon is shadowed in the nuclear medium.

The MCMC cascade has being successfully applied for the interpretation of photonuclear reactions within large ranges of target masses and incident energies. For low energies - typically below few $\mathrm{GeV}$ - the mean free path of the photon is much larger than the nuclear radius, typically hundreds of fermis, and the electromagnetic interaction between the photon and a bound nucleon may take place within the total nuclear volume with equal probability. For higher energies, however, the so called hadronization of the incoming photon takes place, reducing the actual number of nucleons that contribute for the reaction. So, in order to calculate the fraction of nucleons that effectively contribute for the photo-nucleus interaction, one has to evaluate the nuclear transparency to vector mesons (V). Such analysis was performed in this work using the MCMC cascade model and assuming that the photon couples 
with a vector meson $\rho$ (no bare photon component) that impinges on the ${ }^{12} \mathrm{C}$ nuclei. For the nuclear density of ${ }^{12} \mathrm{C}$ we took the shell model distribution from ref. 15 :

$$
\rho(r)=\frac{4}{\left(a_{0} \sqrt{\pi}\right)^{3}}\left(1+\frac{\delta r^{2}}{a_{0}^{2}}\right) \exp \left(\frac{-r^{2}}{a_{0}^{2}}\right)
$$

where $a_{0}=1.65 \mathrm{fm}, \delta=(1 / 6)(A-4)$ with the normalization $A=4 \pi \int \rho(r) r^{2} d r$. The total $\rho-N$ cross section $\sigma_{\rho N}$ is assumed to be equal to the total $\pi^{0}-N$ cross section, which is approximately constant $(\sim 27 \mathrm{mb})$ within 4.0 to $6.0 \mathrm{GeV}$ and is calculated in the MCMC routine. ${ }^{2}$

The nuclear transparency to $\rho$ mesons is calculated as a function of the radial distance $\mathrm{T}(r)$ distributing the impact parameter (b) uniformly in the area of a disc perpendicular to the photon direction and evaluating the number of primary $\rho$-nucleon scatterings. Figure 3 presents the result for the nuclear transparency of $\rho$ mesons for $k$ $=5.2 \mathrm{GeV}$ evaluated in the two halves of the nuclei. $\mathrm{T}_{+}(r)$ corresponds to the transparency in the region $\mathrm{z}<0$ and $T_{-}(r)$ for $\mathrm{z}>0$, where $\mathrm{z}$ is the direction of the incoming vector meson.

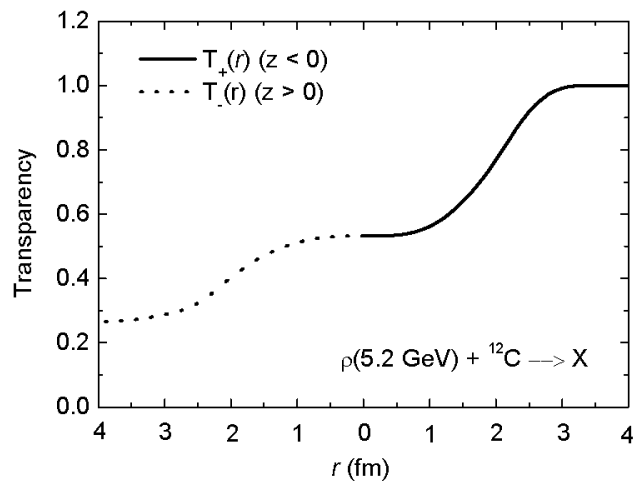

FIGURE 3. Nuclear transparency $\mathrm{T}(r)$ calculated in the MCMC model for a $\rho$ meson of $5.2 \mathrm{GeV}$ impinging on a ${ }^{12} \mathrm{C}$ nucleus. The solid/dotted line represents the corresponding transparency at the forward/backward portion of the nucleus. Details in the text.

So, the total number of nucleons that contribute to the photoproduction mechanism can be written as:

$$
A_{\text {shad }}=4 \pi \int_{0}^{\infty}\left(\frac{\mathrm{T}_{+}(r)+\mathrm{T}_{-}(r)}{2}\right) \rho(r) r^{2} d r=7.163
$$

where, for the case of a bare photon $\left(\mathrm{T}_{+}(r)=\mathrm{T}_{-}(r)=1\right)$, we easily recover the well known condition $A_{\text {shad }}=A$. The ratio $A_{\text {shad }} / A=0.597$ is approximately $30 \%$ lower than some experimental estimates ${ }^{14}(\sim 80 \%)$ and should be further investigated in the future. 


\section{Results}

Since we have taken into account the shadowing effect of the incoming photon, we can calculate the nuclear incoherent (NI) cross section for the photoproduction of neutral pions from ${ }^{12} \mathrm{C}$ using the same prescription outlined in previous works ${ }^{2,6}$, just replacing $A \rightarrow A_{\text {shad }}$. The result for the single differential cross section using the PWIA for the MD is shown in figure 4.

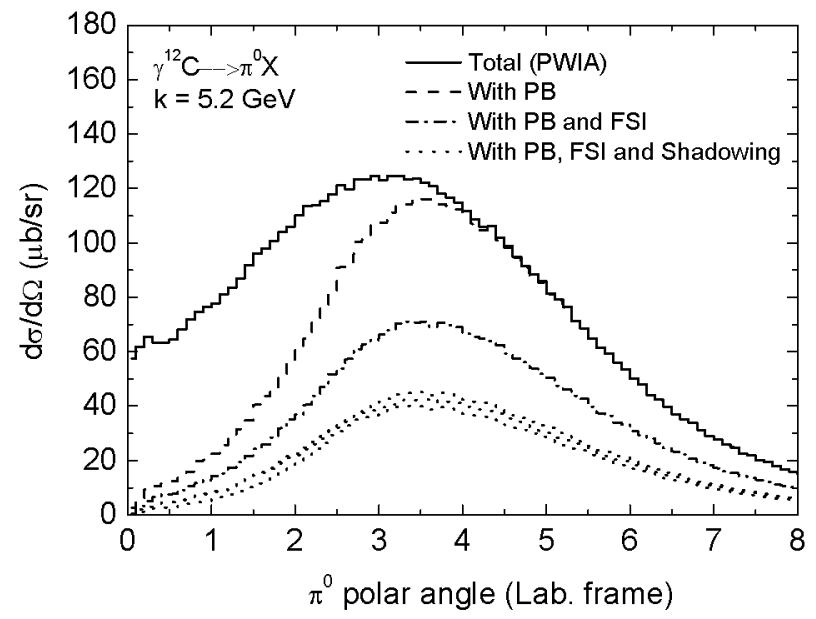

FIGURE 4. Nuclear incoherent cross section for $\pi^{0}$ photoproduction on ${ }^{12} \mathrm{C}$. The solid line is the PWIA, while the dashed line includes the Pauli-blocking suppression factor. The full calculation with/without the nuclear shadowing is represented by the dotted/dashed-dotted lines. Also plotted are the upper and lower limits for the full calculation obtained via the analysis of the sensitivity of the model with respect to the MD parameterizations presented in Table 1 .

As shown in figure 4, the NI cross section is forward peaked since it is proportional to the elementary $\pi^{0}$ production on the nucleon ${ }^{2}$, which present a forward peak behavior. The total cross section, labeled as PWIA, is simply the target mass times the elementary cross section. The Pauli-suppression of the cross section for low momentum transfer is represented by the dashed line, indicating that $\mathrm{N}-\mathrm{N}$ correlations are relevant up to approximately 4 degrees of polar angles for $5.2 \mathrm{GeV}$. The inclusion of FSI of the produced pions reduces the cross section by approximately $40 \%$ (dashed-dotted line). Such reduction can be further investigated by comparing the prediction of other models, such as the well known Glauber model ${ }^{16}$, with the results obtained with the MCMC cascade. The Glauber model consists of a powerful tool to describe the absorption of hadrons in nuclei. This model has been widely accepted but has some limitations as it does not include nuclear correlations and the local density fluctuations. Another disadvantage of the Glauber model is its inability to predict which fraction of the energy of the incident photon is distributed to the bound nucleons during the photoproduction mechanism and at each binary $\pi-N$ scattering. 
The absorption effects in the Glauber model can be calculated by the well known formula:

$$
N_{\text {Glauber }}=\frac{2 \pi}{\sigma_{p N}} \int_{0}^{\infty}\left(1-\exp \left(-\sigma_{p N} \int \rho(b, z)\right)\right) b d b=7.22,
$$

where one immediately finds an absorption of about $40 \%\left(N_{\text {Glauber }} / A \sim 0.6\right)$. This result shows very nicely that two completely different models - the Glauber and the cascade - are consistent with each other in describing attenuation effects of hadrons in the nuclei. On the other hand, the inclusion of the nuclear shadowing in the MCMC cascade yields to a higher attenuation of the nuclear cross section, which can not be tested with the Glauber model as it does not account for such effect.

The double differential cross section including the Pauli-blocking, the mesonnucleus FSI and the shadowing effect is presented in Figure 5 using the PWIA for the MD. The dotted lines indicate the upper and lower limits of the model taking into account different parameterizations for the MD (Table 1).

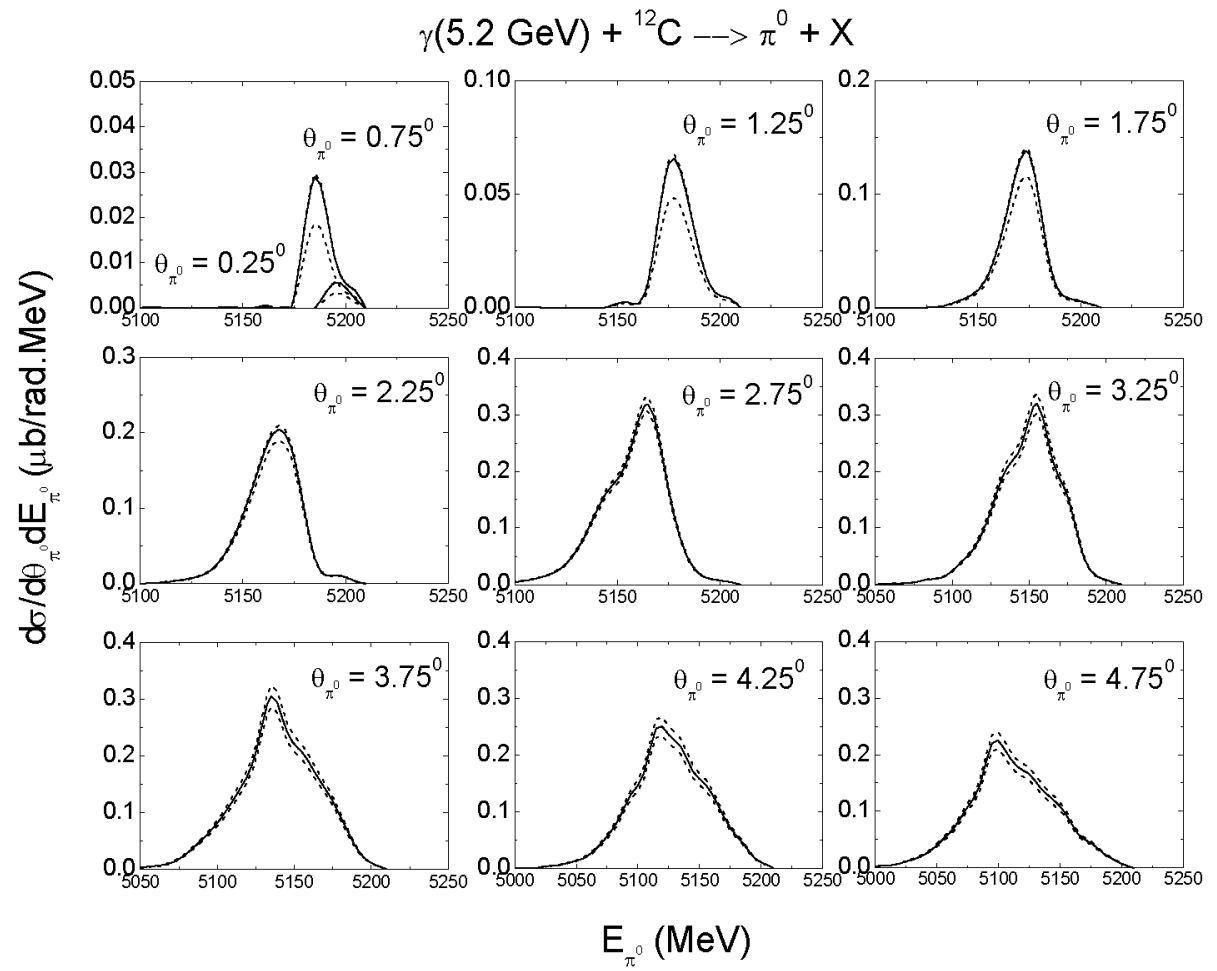

FIGURE 5. Double differential cross section for incoherent $\pi^{0}$ photoproduction on $12 \mathrm{C}$. The dotted lines indicate the uncertainty of the MCMC model associated with the MD. 


\section{CONCLUSIONS AND FINAL REMARKS}

We have presented an extended version of the MCMC model suitable for calculations of the nuclear incoherent cross section of $\pi^{0}$ photoproduction from ${ }^{12} \mathrm{C}$. A detailed analysis of the MD of the bound nucleons was carried out taking into account a huge amount of the worldwide quasi-free 1-s and 1- $p$ proton knock-out data.

The shadowing effect on the total photo-nucleus cross section was evaluated using the MCMC algorithm for the transport of $\rho$ mesons in the nuclear matter and evaluating the nuclear transparency. The NI single and double differential cross sections were re-calculated using the same prescriptions of previous works and also including the hadronization of the incoming photon. The FSI effects of the produced pions were taken into account using the MCMC formalism, where we found an attenuation of approximately $40 \%$ due to absorption and re-scattering. Such result showed that the cascade and Glauber approaches are mutually consistent for the description of the attenuation of hadrons in nuclei.

\section{ACKNOWLEDGMENTS}

One of the authors (TER) thanks the financial support from the Brazilian Agency FAPESP and for the pleasant and fruitful attendance of the VII Latin American Symposium of Nuclear Physics and Applications.

\section{REFERENCES}

1. http://www.jlab.org/primex/primex_notes/PR99-014.ps (PrimEx Proposal).

2. T. E. Rodrigues, J. D. T. Arruda-Neto, J. Mesa, C. Garcia, K. Shtejer, D. Dale, and I. Nakagawa Phys. Rev. C 71, 051603(R) (2005).

3. T. E. Rodrigues, J. D. T. Arruda-Neto, A. Deppman, V. P. Likhachev, J. Mesa, C. Garcia, K. Shtejer, G. Silva, S. B. Duarte, and O. A. P. Tavares, Phys. Rev. C 69, 064611 (2004).

4. T. E. Rodrigues, M. N. Martins, C. Garcia, J. D. T. Arruda-Neto, J. Mesa, K. Shtejer, and F. Garcia, Phys. Rev. C 75, 014605 (2007).

5. B. Krusche et al., Eur. Phys. J. A 22, 277 (2004).

6. T. E. Rodrigues, J. D. T. Arruda-Neto, J. Mesa, C. Garcia, K. Shtejer, D. Dale, and I. Nakagawa, Brazilian Journal of Physics 36, 4B 1366 (2006).

7. T. E. Rodrigues, J. D. T. Arruda-Neto, J. Mesa, C. Garcia, K. Shtejer, D. Dale, I. Nakagawa, and P. Cole, " $\eta-\rightarrow \gamma \gamma$ decay width via the Primakoff cross section," submitted to Phys. Rev. Lett. (2007).

8. A. Browman et al., Phys. Rev. Lett. 32, 1067 (1974).

9. L. Lapikás, G. van der Steenhoven, L. Frankfurt, M. Strikman, and M. Zhalov, Phys. Rev. C 61, $064325(2000)$.

10. J. Mougey, et al., Nucl. Phys. A262, 461 (1976).

11. M. Bernheim et al., Nucl. Phys. A375, 381 (1982).

12. K. Nakamura, S. Hiramatsu, T. Kamae, H. Muramatsu, N. Izutsu, and Y. Watase, Nucl. Phys. A268, $381(1976)$.

13. G. van der Steenhoven, H. P. Blok, E. Jans, M. De Jong, L. Lapikás, E. N. M. Quint, and P. K. A. De Witt Huberts, Nucl. Phys. A480, 547 (1988).

14. T. H. Bauer, R. D. Spital, D. R. Yennie, and F M. Pipkin, Rev. Mod. Phys. 50, 261 (1978).

15.W. T. Meyer, A. Browman, K. Hanson, A. Osborne, A. Silverman, and F. E. Taylor, Phys. Rev. Lett. 28, 1344 (1972).

16. G. Faldt, Nucl. Phys. 43, 591 (1972). 
Copyright of AIP Conference Proceedings is the property of American Institute of Physics and its content may not be copied or emailed to multiple sites or posted to a listserv without the copyright holder's express written permission. However, users may print, download, or email articles for individual use. 